DEPARTAMENTO DE QUIMICA ORGÃNICA E BIOLOGICA

Diretor: Prof. Fonseca Ribeiro

DEPARTAMENTO DE MICROBIOLOGIA E IMUNOLOGIA

Diretor: Prof. Interino Paulo M. G. de Lacerda Junior

\title{
NEUTRALIZAÇÃO DA TOXINA TETÂNICA PELA CLOROFILA HIDROSSOLÜVEL
}

\author{
(NEUTRALIZATION OF TETANIC TOXINE BY HYDRO-SOLUBLE \\ CHLOROPHYLL)
}

Fonseca Ribeiro

Paulo M. G. Lacerda Jr.

Em 1942, Fonseca Ribeiro e Guimarães ( ${ }^{1}$ ) apresentaram suas primeiras observações sôbre a neutralização da toxina tetânica e do veneno crotálico, pelo clorifilinato de potássio.

Para a toxina tetânica, verificaram uma proteção contra 3 D.M.M., injetando a toxina misturada com solução aquosa de clorofilinato de potássio, admitindo também a possibilidade de permanência do poder antigênico.

Procurando ampliar essas observações, resolvemos verificar:

a) Se uma toxina tetânica poderia ser desintoxicada "in vitro" e em que tempo.

b) Se na desintoxicação "in vitro" a toxina seria destruida ou sòmente neutralizado sem poder tóxico, conservando-se o caráter antigênico.

\section{PARTE EXPERIMENTAL}

Para verificar quantas D.M.M. seriam neutralizadas por uma quantidade fixa de clorofila, utilizamos cobaias de 350 gramas. Partindo de uma toxina sêca, foi preparada uma solução em glicerina a $50 \%$, a qual diluiu-se de modo a conter $10,100,500,750$ e 1000 D.M.M. em um volume de $0,75 \mathrm{~cm} 3$; juntou-se, a cada uma dessas doses, $0,5 \mathrm{~cm} 3$ de uma solução a $1 \%$ de clorofila hidrossolúvel de May and Baker Ltd.. Após contacto de 15 minutos, ao abrigo da luz, os animais eram injetados com a mistura, por via intramuscular.

Com a mesma toxina, foi feita uma diluição, sem clorofila, de modo a conter 1 D.M.M., com a qual foram injetadas as cobaias testemunhas. Os resultados obtidos estão expressos no quadro I. 
Rev. Fac. Med. Vet. S. Páulo - Vol. 4, fasc. 2, 1950

QUADRO I

Ação desintoxicante da clorifila sôbre a toxina tetânica

\begin{tabular}{|c|c|c|c|c|c|}
\hline \multirow{2}{*}{$\begin{array}{l}\text { Cobaia de } \\
350 \mathrm{~g}\end{array}$} & \multirow{2}{*}{ D.M.M. } & \multicolumn{4}{|c|}{ Resultado } \\
\hline & & 24 horas & 48 horas & 72 horas & 96 horas \\
\hline 1 & 10 & 0 & 0 & 0 & 0 \\
\hline 2 & 10 & 0 & $Q$ & 0 & 0 \\
\hline 3 & 100 & 0 & 0 & 0 & 0 \\
\hline 4 & 100 & 0 & 0 & 0 & 0 \\
\hline 5 & 500 & 0 & 0 & 0 & 0 \\
\hline 6 & 500 & 0 & 0 & 0 & $\mathrm{~S}$ \\
\hline 7 & 500 & 0 & 0 & 0 & $\mathbf{S}$ \\
\hline 8 & 500 & 0 & 0 & 0 & 0 \\
\hline 9 & 500 & 0 & 0 & 0 & 0 \\
\hline 10 & 500 & 0 & 0 & 0 & 0 \\
\hline 11 & 500 & 0 & 0 & 0 & 0 \\
\hline 12 & 750 & s & $\mathrm{S}$ & $S$ & + \\
\hline 13 & 750 & $\mathbf{S}$ & $\mathbf{s}$ & $\mathbf{S}$ & + \\
\hline 14 & 750 & $\mathbf{s}$ & S & $\mathbf{S}$ & + \\
\hline 15 & 750 & $\mathbf{S}$ & $\mathbf{s}$ & $\mathbf{s}$ & + \\
\hline 16 & 750 & $\mathbf{s}$ & $s$ & $\mathbf{s}$ & + \\
\hline 17 & 1000 & S & $\mathrm{S}$ & $-t$ & \\
\hline 18 & 1000 & $\mathbf{S}$ & + & & \\
\hline 19 & 1000 & $\mathbf{S}$ & $\mathbf{S}$ & + & \\
\hline 20 & 1000 & S & $\dashv$ & & \\
\hline 21 & 1000 & $\mathbf{s}$ & + & & \\
\hline 22 & & $\mathbf{S}$ & s & S & + \\
\hline 23 & & $\mathrm{~S}$ & $\mathrm{~S}$ & $\mathbf{S}$ & $-1-$ \\
\hline 24 & Testemunhos & S & $\mathbf{s}$ & $\mathbf{S}$ & $t$ \\
\hline 25 & (1 D.M.M.) & $\mathrm{S}$ & $\mathrm{s}$ & $\mathbf{s}$ & $-t$ \\
\hline 26 & & $\mathrm{~s}$ & $\mathbf{S}$ & $\mathbf{S}$ & + \\
\hline 27 & & $\mathbf{S}$ & $\mathrm{s}$ & 3 & + \\
\hline
\end{tabular}

Nota: $\mathbf{0}=$ ausência de sintomas; $\mathbf{S}=$ sintomas de tétano; + morte.

Verifica-se, por êste quadro, que $0,5 \mathrm{~cm} 3$ da solução aquosa de clorofilinato de potássio a $1 \%$ foi suficiente para neutralizar o efeito tóxico de 500 D.M.M. 
de toxina tetânica; para 750 D.M.M., o efeito protetor da clorofila não mais se manifestou, nas meșmas condições.

Tendo consignado êstes resultados, decidimos verificar a possibilidade de desintoxicar uma toxina tetânica, pelo contacto com clorofila hidrossolúvel, em estufa.

Tomamos então $100 \mathrm{~cm} 3$ de toxina tetânica (D.M.M. para cohaia $=1 / 15.000$ ) e adicionamos 1 grama de clorofila hidrossolúvel, prèviamente esterilizada $\mathrm{em}$ autoclave, por 20 minutos, a $115^{\circ} \mathrm{C}$.

Essa mistura de toxina com clorofila foi conservada em estufa a $37^{\circ} \mathrm{C}$ e amostras dírias eram tiradas para acompanhar a desintoxicação. Depois de têrmos obtido a desintoxicação de mais de uma partida, verificamos que a desintoxicação total era obtida após uma permanência de 8 a 10 dias, na estufa.

A toxina tetânica assim tratada, inoculada em cobaias de 350-400 gramas, na dose de $1 \mathrm{~cm} 3$, não mais foi capaz de provocar sinais de tétano.

Obtido assim um número razoável de animais injetados nessas condições, 30 dias ajós, mais ou menos, receberam os mesmos de 5 a 10 D.M.M., injetadas por via subcutânea, cujos resultados estão expressos no quadro II.

\section{QUADRO II}

Conservação do poder antigênico da mistura toxina tetânica e clorofila hidrossolńveì

\begin{tabular}{|c|c|c|c|c|}
\hline \multirow{2}{*}{$\begin{array}{l}\text { Cobaias injeta- } \\
\text { das com } 1 \mathrm{~cm} 3 \\
\text { de toxina-cloro } \\
\text { fila }\end{array}$} & \multicolumn{2}{|c|}{$\begin{array}{c}\text { Reinjetadas com } 5 \text { D.M.M. } \\
\text { (19 cobaias) }\end{array}$} & \multicolumn{2}{|c|}{$\begin{array}{l}\text { Reinjetadas com } 10 \text { D.M.M. } \\
\text { (13 cobaias) }\end{array}$} \\
\hline & sobrevivem & inorrem & sobrevivem & morrem \\
\hline 32 & 13 & 6 & 6 & 7 \\
\hline
\end{tabular}

4 cobaias testemunhas injetadas com 1 D.M.M. morrem em 96 horas

fstes resultados indicam que, dos animais que receberam a injeção de toxina tetânica desintoxicada pela clorofila hidrossolúvel, $68,4 \%$ resistiram à subseqüente inoculação de 5 D.M.M. e 46,1\% resistiram a 10 D.M.M..

Com o fim de verificar se a desintoxicação da toxina tetânica pela clorofila poderia depender mais do fator luz do que do fator temperatura (estufa a $37^{\circ} \mathrm{C}$, escuro), foram preparados dois frascos com $0.5 \mathrm{~g}$ de clorofila e $100 \mathrm{~cm} 3$ de toxina tetânica $(0,25 \mathrm{~cm} 3$ da diluição a $l: 50.000=1$ D.M.M. camundongo $)$. Um dos frascos foi conservado na estufa a $37^{\circ} \mathrm{C}$ (escuro) e o outro foi mantido na luminosidade e temperatura ambientes. 
De ambos os frascos foram retiradas amostras em tempos sucessivos, a fim de testar a toxicidade em camundongos, como se vê no quadro III.

\section{QUADiso III}

Comparação da ação desintoxicante da clorofila sôbre a toxina tetânica na luz em condições ambiente, e 10 escuro a $37 \circ \mathrm{C}$ (as doses injetadas em cada animal foram $0,25 \mathrm{~cm} 3$ das diluições correspondentes)

\begin{tabular}{|c|c|c|c|c|c|c|c|}
\hline Tempo de & $\begin{array}{r}\text { N.o de o } \\
\text { an }\end{array}$ & $\begin{array}{l}\text { dem dos } \\
\text { mais }\end{array}$ & & & Sobrevida & los anima & \\
\hline $\begin{array}{c}\text { contacto da } \\
\text { mistura }\end{array}$ & $\begin{array}{c}\text { Material } \\
\text { da } \\
\text { estufa }\end{array}$ & $\begin{array}{c}\text { Material } \\
\text { em am- } \\
\text { biente }\end{array}$ & Diluição & 24 horas & 48 horas & 72 horas & 96 horas \\
\hline 2 horas & $\begin{array}{r}1 \\
2 \\
- \\
-\end{array}$ & $\begin{array}{r}- \\
- \\
4\end{array}$ & 1:50.000 & $\begin{array}{l}0 \\
0 \\
0 \\
0\end{array}$ & $\begin{array}{l}\mathbf{S} \\
\mathbf{S} \\
\mathbf{S} \\
\mathbf{S}\end{array}$ & $\begin{array}{l}\mathbf{S} \\
\mathbf{S} \\
\mathbf{S} \\
\mathbf{S}\end{array}$ & $\begin{array}{l}+ \\
+ \\
+ \\
+\end{array}$ \\
\hline 24 horas & $\begin{array}{r}5 \\
6 \\
7 \\
8 \\
- \\
- \\
- \\
-\end{array}$ & $\begin{array}{r}- \\
- \\
- \\
- \\
9 \\
10 \\
11 \\
12\end{array}$ & $\begin{array}{l}1: 50.000 \\
1: 50.000 \\
1: 40.000 \\
1: 40.000 \\
1: 50.000 \\
1: 50.000 \\
1: 40.000 \\
1: 40.000\end{array}$ & $\begin{array}{l}0 \\
0 \\
0 \\
0 \\
0 \\
0 \\
0 \\
0\end{array}$ & $\begin{array}{l}0 \\
0 \\
0 \\
0 \\
\mathrm{~S} \\
\mathrm{~S} \\
\mathrm{~S} \\
\mathrm{~S}\end{array}$ & $\begin{array}{l}0 \\
0 \\
0 \\
0 \\
\mathrm{~S} \\
\mathrm{~S} \\
\mathrm{~S} \\
\mathrm{~S}\end{array}$ & $\begin{array}{l}0 \\
0 \\
0 \\
0 \\
+ \\
+ \\
+ \\
+\end{array}$ \\
\hline 48 horas & $\begin{array}{l}13 \\
14 \\
15 \\
16 \\
- \\
- \\
- \\
-\end{array}$ & $\begin{array}{l}- \\
- \\
- \\
- \\
17 \\
18 \\
19 \\
20\end{array}$ & $\begin{array}{l}1: 30.000 \\
1: 30.000 \\
1: 20.000 \\
1: 20.000 \\
1: 50.000 \\
1: 50.000 \\
1: 40.000 \\
1: 40.000\end{array}$ & $\begin{array}{l}0 \\
0 \\
0 \\
0 \\
0 \\
0 \\
0 \\
0\end{array}$ & $\begin{array}{l}0 \\
0 \\
0 \\
0 \\
0 \\
0 \\
\mathrm{~S} \\
\mathrm{~S}\end{array}$ & $\begin{array}{l}0 \\
0 \\
0 \\
0 \\
S \\
S \\
S \\
S\end{array}$ & $\begin{array}{l}0 \\
0 \\
0 \\
0 \\
S \\
S \\
S \\
S\end{array}$ \\
\hline
\end{tabular}

Nota: $0=$ ausência de sintomas; $\mathbf{S}=$ sintomas de tétano; + morte.

O quadro III evidencia que a desintoxicação da toxina telânica pela clorofila hidrossolível se processa com mais velocidade na mistura colocada a $37^{\circ} \mathrm{C}$ no escuro do que na mistura mantida na luz e temperatura ambientes. $E^{*}$ assim que se verifica que, com 48 horas de contacto, a mistura conservada a $37^{\circ} \mathrm{C}$. já não mostrava toxicidade a $1: 20.000$, enquanto no mesmo espaço de tempo, a mistura deixada no ambiente ainda possuia efeitos tóxicos na diluição a $1: 50.000$. 


\section{DISCUSSÃO}

A desintoxicação de toxinas microbianas, assim como de venenos ofídicos por intermédio de várias substâncias dotadas de ação fotoquímica, tem sido am. plamente descrita na literatura; as substâncias tóxicas, perdendo o caráter letal, perdem também a propriedade antigênica. A simples irradiação dos venenos de cobra determina a destruição dos mesmos, como foi demonstrado por ArTHUS $\left(^{(2}\right)$, talvez pela coexistência, nestes venenos, de substâncias fluorescentes, conforme veficaram TABORDA e TABORDA $\left({ }^{3}\right)$. Um detalhe interessante, no particular, foi acrescentado por Lidinow $\left({ }^{4}\right)$; trabalhando com o veneno de naja, observou que sua destruiçáa só ocorre nos comprimentos de onda compreendidos entre 300 e $2.800 \mathrm{~A}$, a não ser que a solução do veneno seja misturada com eosina, antes da irradiação, passando então a ser destruido com maiores comprimentos de onda.

Para todos êsses casos, como também nas verificações de Shorte e MaLLICK $\left(^{5}\right)$ com o azul de metileno, existe necessidade da irradiação do veneno puro ou misturado com a substância fotossensibilizadora, para que haja destruição do poder tóxico, destruindo-se também o poder antigênico.

Nas nossas experiências com a clorofila hidrossolúvel, o fenômeno se passa de maneira diferente, não só porque não há necessidade de irradiação da mistura toxina-clorofila, como também porque a toxina desintoxicada conserva ainda a propriedade antigênica. Não se pôde, até o presente, estabelecer um paralelo entre a toxina desintoxicada pela clorofila e a anatoxina (desintoxicaçáo pelo aldeído fórmico), mas náo resta dúvida em que o processo da clorofila é muito mais rápida, exigindo aproximadamente um terço do tempo necessário para a obtenção da anatoxina.

\section{RESUMO}

Confirmando o trabalho de Fonseca Ribeiro e Guimarães, os AA. demonstraram mais que, com 15 minutos de contacto, $0,5 \mathrm{~cm} 3$ de clorofila hidrossolúvel a $1 \%$ neutraliza 500 D.M.M. de toxina tetânica; que $100 \mathrm{~cm} 3$ de toxina tetânica ( $1 \mathrm{~cm} 3=15.000$ D.M.M. cobaia) em contacto com $1,0 \mathrm{~g}$ de clorofila hidrossolúvel, na esufa a $37^{\circ} \mathrm{C}$, perde totalmente sua toxicidade, ao cabo de 8-10 dias, conservando o poder antigênico: 32 cobaias injetadas com 1 cm3 cada, da toxina desintoxicada pela clorofila, são injetadas 30 dias depois, com 5 e 10 D.M.M. de toxina, sobrevivendo, respectivamente, $68,4 \%$ e $46,1 \%$; demonstraram ainda que o fator luz é menos importante do que o fator temperatura (estufa $37^{\circ} \mathrm{C}$ ) na desintoxicação da toxina tetânica pela clorifila hidrossolúvel. 


\section{SUMMARY}

Confirming the studies of FonseCA RiBEIRo and GUimarãEs, the authors showed besides that with a contact for 15 minutes, $0.5 \mathrm{ml}$. of hydro-soluble chlorophyll at $1 \%$ neutralizes 500 M.L.D. of tetanic toxine; that $100 \mathrm{ml}$. of tetanic toxine (concentration of $1 \mathrm{mll}=15,000$ M.L.D. guinea pigs) in contact with $1,0 \mathrm{~g}$. of hydro-soluble chlorophyll in the incubator at $37^{\circ} \mathrm{C}$. loses completely its toxicity in from 8 to 10 days, while still keeping its antigenic quality: 32 guinea pigs injected with $1 \mathrm{ml}$. each of this toxin, detoxicated by chlorophyll, were injected 30 days after with 5 and 10 M.L.D. of toxine, and $68.1 \%$ and $16.1 \%$, respectively, survived; the light factor is of less importance than the lemperature factor (incubator $37^{\circ} \mathrm{C}$. .) in the detoxication of tetanic toxine by the hydro-soluble chlorophyll process.

\section{BIBLIOGRAFIA}

1 - Fonsica Rimeno, D. e Grmmanazes, 1. M. - 1942 - Estuhlo sôhre a ação antitúxica da clorofila: P'rimeiros resultados com o veneno crotálico (Crotalus terrificus terrificus) e toxina tetânica - Rev. Fac. Med. Vet., S. Panlo, 2, 3-11.

2 - Antrus, A. - 1930 - Le venin de cobra rendu inoffensif par l'action des rayons ultra-violets a perdu son pouvoir immunisant. Compt. Rend. Soc. Biol., 103, 130-2

3 - Tamorda, A. R. e TAborda, L. C. - 1941 - Da relação entre o corante dos venenos de cobra e a sua fluorescência. Mem. Inst. Butantan, 15, 47-5.5

4 - Eidinow, $\Lambda$. - 1930 - The effect of irradiation on cobra venon and antivenin. British Jour. Exp. Path., 11, 61-72

5 - Shroptr, H. E. and Mallick, S. M. K. - 1935 - Detoxication of snake venon by the photodynamic action of methylene blue. Ind. Jour. Med. Res., 22, 529-36 